\title{
Natural frequencies of curved elastic arcs
}

\author{
N. J. Kudva, A. H. Nayfeh, and M. P. Kamat \\ Virginia Polytechnic Institute and State University, Blacksburg, Virginia 24061 \\ (Received 20 March 1978; revised 26 July 1978)
}

\begin{abstract}
A perturbation analysis is presented for calculating the inextensional natural frequencies of curved elastic arcs. Variation of the radius of curvature along the arc length is accounted for by considering the curvature to be a perturbation from a constant curvature, and utilizing the method of strained parameters. Frequencies thus derived for hinged parabolic arcs demonstrate good agreement with finite element solutions. The analysis could easily be extended to determine the natural frequencies of noncircular curved plates and shells.
\end{abstract}

PACS numbers: $43.20 . \mathrm{Mv}, 43.40 . \mathrm{Cw}$

\section{INTRODUCTION}

Natural frequencies of curved elastic arcs have been determined by several authors. Volterra and Morell ${ }^{1}$ derived approximate formulas for various hinged elastic arcs, including the effect of axial stretching but ignoring the variation of curvature. Romanelli and Laura ${ }^{2}$ took into account the variation of curvature as a function of the arc length and obtained results slightly different from the results of Volterra and Morell. Both the above analyses used the Rayleigh-Ritz method to obtain the fundamental frequencies. In contrast, the present investigation utilizes the method of strained parameters to account for variable curvature.

A description of the method of strained parameters is given by Nayfeh. ${ }^{3}$ This method is frequently used to determine approximate solutions of weakly nonlinear differential equations and linear differential equations with periodic coefficients. The application of the method of strained parameters to the present problem is detailed below,

The translational and rotational equations of motion can easily be derived by considering the equilibrium of the beam element shown in Fig. 1. They are as follows:

$$
\begin{aligned}
& \frac{\partial}{\partial s^{\prime}}\left(n^{\prime} \cos \theta-q^{\prime} \sin \theta\right)=\rho A \frac{\partial^{2} u^{\prime}}{\partial t^{\prime 2}}, \\
& \frac{\partial}{\partial s^{\prime}}\left(n^{\prime} \sin \theta+q^{\prime} \cos \theta\right)=\rho A \frac{\partial^{2} w^{\prime}}{\partial t^{\prime 2}}, \\
& q^{\prime}+\frac{\partial m^{\prime}}{\partial s^{\prime}}=\rho I \frac{\partial^{2} \theta}{\partial t^{\prime 2}} .
\end{aligned}
$$

The moment displacement relation from beam theor $y^{4}$ is

$$
m^{\prime}=E I\left(\frac{\partial \theta}{\partial s^{\prime}}+\frac{w^{\prime}}{R^{\prime 2}}\right),
$$

where $n^{\prime}$ is the axial force, $q^{\prime}$ is the force component normal to $n^{\prime}, m^{\prime}$ is the bending moment, $I$ is the moment of inertia of the cross section, $\rho$ is the mass density, $A$ is the area of cross section, and $R^{\prime}$ is the radius of curvature expressed as a function of the length $s^{\prime}$. From geometric considerations we can write

$$
\begin{aligned}
& \sin \theta=\frac{\partial w^{\prime}}{\partial s^{\prime}}\left[\left(1+\frac{\partial u^{\prime}}{\partial s^{\prime}}\right)^{2}+\left(\frac{\partial w^{\prime}}{\partial s^{\prime}}\right)^{2}\right]^{-1 / 2}, \\
& \cos \theta=\left(1+\frac{\partial u^{\prime}}{\partial s^{\prime}}\right)\left[\left(1+\frac{\partial u^{\prime}}{\partial s^{\prime}}\right)^{2}+\left(\frac{\partial w^{\prime}}{\partial s^{\prime}}\right)^{2}\right]^{-1 / 2} .
\end{aligned}
$$

We consider only the linear problem; that is we do not account for axial stretching. Hence we need to consider only transverse equilibrium, Eqs. (2) and (3). For simplicity we also consider no applied axial loads although this presents no additional difficulty.

Following Nayfeh, ${ }^{5}$ we nondimensionalize the governing differential equations using the following nondimensional variables:

$$
\begin{gathered}
s=\frac{s^{\prime}}{L} ; \quad w=\frac{w^{\prime} A L}{I} ; \quad u=\frac{A^{2} L^{3} u^{\prime}}{I^{2}} ; t=t^{\prime}\left(\frac{E I}{\rho A L^{4}}\right)^{1 / 2}=t^{\prime} T ; \\
R=\frac{R^{\prime}}{L} .
\end{gathered}
$$

Substituting the above relations in Eqs. (3)-(5), we obtain

$$
\begin{aligned}
& \sin \theta=\epsilon w_{, s}+N L T \\
& \cos \theta=1+N L T \\
& \theta=\epsilon w_{, s}+N L T \\
& m=\epsilon E I L^{-1}\left(w_{, s s}+R^{-2} w\right)+N L T \\
& q=\epsilon\left[\frac{\rho I}{T^{2}} w_{, s t t}-\frac{E I}{L^{2}} w_{, s s s}-E I\left(\frac{w}{R^{2}}\right)_{, s}\right]+N L T,
\end{aligned}
$$

where a comma indicates differentiation, $N L T$ represents nonlinear terms which are neglected, and $\epsilon=I / A L^{2}$ is a nondimensional parameter.

In the above process of nondimensionalization the parameter $\epsilon$ naturally falls out as a coefficient which decides the degree of nonlinearity. Another nondimensionalization scheme may give rise to another such pa-

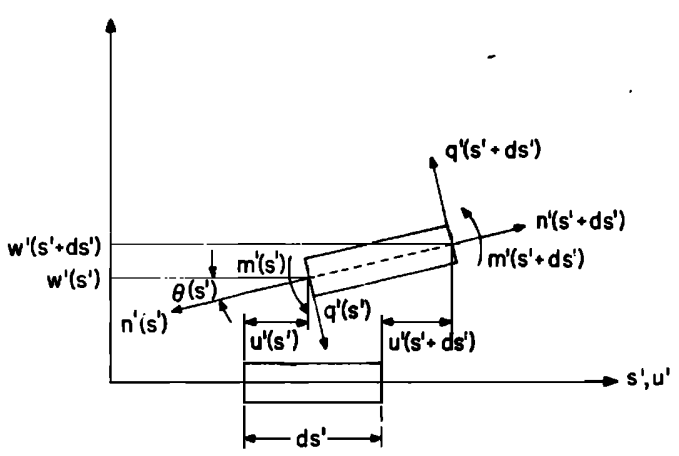

FIG. 1. Schematic of a beam element. 
TABLE I. Comparison of the exact and approximate frequencies for three circular arcs.

Nondimensional frequency $\omega$

\begin{tabular}{lcc}
$R$ & Exact [Eq. (19)] & Perturbation soln. \\
\hline 1.172 & 9.463 & 9.452 \\
1.272 & 9.548 & 9.509 \\
1.372 & 9.633 & 9.554 \\
\hline \hline
\end{tabular}

rameter. For most arches this quantity which represents the square of the slenderness ratio is very small. Hence, this parameter is chosen as the perturbation parameter in the present analysis. This choice is by no means unique but is incidental to the nondimensionalization scheme and is appropriate by virtue of its magnitude. Furthermore, the first-order corrections to frequency due to the effect of variation in the radius of curvature turn out to be independent of the perturbation parameter and hence it need not be directly identifiable.

Using Eq. (6), we can write the linearized version of Eq. (2) in nondimensional form as

$$
w_{, t t}+w_{, s s s}+\left(w R^{-2}\right)_{, s s}=\epsilon w_{, s s t} \cdot
$$

The term on the right-hand side of Eq. (7) represents the effect of rotary inertia. We express the radius of curvature as a deviation from a constant as

$$
R(s)=R_{0}+\epsilon R_{1}(s) .
$$

Hence, $R_{, s}=\epsilon R_{1, s}$ and $R_{\text {ss }}=\epsilon R_{1, s s}$. We require $\epsilon R_{1}(s)$ to be "small" in some sense compared with $R_{0}$ and this influences the accuracy of the perturbation approximation. This is exemplified in the numerical results. Substituting Eq. (8) into Eq. (7) and retaining terms up to $O(\epsilon)$, we obtain

$$
\begin{aligned}
w_{, t t} & +w_{, s s s}+R_{0}^{-2} w_{, s s} \\
& =\epsilon\left[w_{, s s t t}+R_{0}^{-3}\left(2 R_{1} w_{, s s}+4 w_{, s} R_{1, s}+2 w R_{1, s s}\right)\right] .
\end{aligned}
$$

\section{SOLUTION OF THE GOVERNING EQUATION AND THE METHOD OF STRAINED PARAMETERS}

We seek a solution of Eq. (9) which is separable in space and time of the form

$$
w(s, t)=\phi(s) \exp (i \omega t) .
$$

Substituting Eq. (10) into Eq. (9), we obtain,

$$
\begin{aligned}
\phi, s s s s & +R_{0}^{-2} \phi_{, s s}-\omega^{2} \phi \\
= & \epsilon\left[-\omega^{2} \phi_{, s s}+R_{0}^{-3}\left(2 R_{1} \phi_{, s s}+4 R_{1, s} \phi_{, s}+2 R_{1, s s} \phi\right)\right] .
\end{aligned}
$$

We seek expansions for $\phi(s)$ and $\omega$ in the form

$$
\begin{aligned}
& \phi(s)=\phi_{0}(s)+\epsilon \phi_{1}(s)+\cdots \\
& \omega=\omega_{0}+\epsilon \omega_{1}+\cdots .
\end{aligned}
$$

Substituting Eqs. (12) into Eq. (11) and equating coefficients of like powers of $\epsilon$, we obtain

Order $\epsilon^{0}$

$$
\phi_{0, s s s}+R_{0}^{-2} \phi_{0, s s}-\omega_{0}^{2} \phi_{0}=0 ;
$$

Order $\epsilon^{1}$

$$
\begin{aligned}
& \phi_{1, s s s s}+R_{0}^{-2} \phi_{1, s s}-\omega_{0}^{2} \phi_{1} \\
& \quad=2 \omega_{0} \omega_{1} \phi_{0}-\omega_{0}^{2} \phi_{0, s s}+R_{0}^{-3}\left(2 R_{1} \phi_{0, s s}+4 R_{1, s} \phi_{0, s}+2 R_{1, s s} \phi_{0}\right) .
\end{aligned}
$$

The general solution of Eq. (13) can be written as

$$
\phi_{0}(s)=c_{1} \sinh \alpha s+c_{2} \cosh \alpha s+c_{3} \sin \beta s+c_{4} \cos \beta s,
$$

where

$$
\alpha^{2}=\left(\omega_{0}^{2}+\frac{1}{4} R_{0}^{-4}\right)^{1 / 2}-\frac{1}{2} R_{0}^{-2} \quad, \quad \beta^{2}=\left(\omega_{0}^{2}+\frac{1}{4} R_{0}^{-4}\right)^{1 / 2}+\frac{1}{2} R_{0}^{-2} .
$$

For specific boundary conditions, we can determine three of the constants $c_{1}, c_{2}, c_{3}$, and $c_{4}$ in terms of the fourth. For simplicity we consider only simply supported arches. Then,

$$
w(s)=w_{\text {,ss }}+R^{-2} w=0 \text { at } s=0 \text { and } 1 .
$$

Substituting Eq. (12) into Eq. (16) and equating coefficients of like powers of $\epsilon$, we obtain,

$$
\begin{aligned}
& \phi_{0}=\phi_{0, s s}=0 \text { at } s=0 \text { and } 1, \\
& \phi_{1}=\phi_{1, s s}=0 \text { at } s=0 \text { and } 1 .
\end{aligned}
$$

From Eqs. (15) and (17) we obtain

$$
\omega_{0}=n \pi\left(n^{2} \pi^{2}-R_{0}^{-2}\right)^{1 / 2} ; \phi_{0}=A \sin n \pi s,
$$

where $A$ is an arbitrary constant. Similar solutions can be obtained for other boundary conditions.

We now consider the first-order problem, Eqs. (14) and (18). We note that their homogeneous counterparts have a nontrivial solution. Hence, the inhomogeneous problem will have a solution if, and only if, a solvability condition is satisfied. To determine the solvability condition, we multiply Eq. (14) by a function $\psi(x)$ that will be specified later, integrate the result by parts from $s=0$ to $s=1$ to transfer the derivatives from $\phi_{1}$ to $\psi$, use Eq. (18), and obtain

$$
\begin{aligned}
& \int_{0}^{1}\left(\psi, s s s s+R_{0}^{-2} \psi_{, s s}-\omega_{0}^{2} \psi\right) \phi_{1} d s+\left[\phi_{1, s s s} \psi+\phi_{1, s} \psi_{, s s}\right]_{s=0}^{s=1} \\
& =\int_{0}^{1}\left[2 \omega_{0} \omega_{1} \phi_{0}-\omega_{0}^{2} \phi_{0, s s}+R_{0}^{-3}\left(2 R_{1} \phi_{0, s s}\right.\right. \\
& \left.\left.\quad+4 R_{1, s} \phi_{0, s}+2 R_{1, s s} \phi_{0}\right)\right] \psi d s .
\end{aligned}
$$

We choose $\psi$ to be a solution of the adjoint homogeneous problem; that is,

$$
\begin{aligned}
& \psi_{, s s s s}+R_{0}^{-2} \psi_{, s s}-\omega_{0}^{2} \psi=0 \\
& \psi=\psi_{, s s}=0 \text { at } s=0 \text { and } 1 .
\end{aligned}
$$

Hence, when $\omega_{0}$ is given by Eq. (19), $\psi=b \sin n \pi s$, where $b$ is a constant. Substituting for $\psi$ into Eq. (20) and solv-

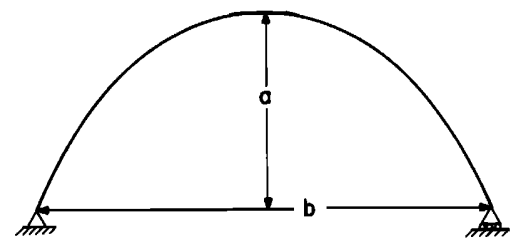

FIG. 2. Schematic of a parabolic arc: (1) $a / b=\frac{1}{3}$, (2) $a / b=\frac{1}{4}$. 
TABLE II. Comparison of the exact and approximate frequencies of two parabolic arcs.

\begin{tabular}{llllll}
\hline \hline & $R_{\max }$ & $R_{\min }$ & $\operatorname{Max}\left|\frac{\epsilon R_{1}(s)}{R_{0}}\right|$ & $\begin{array}{c}\text { Nondimensional frequency } \omega \\
\text { Perturbation finite-element }\end{array}$ \\
\hline $\begin{array}{l}\text { (Zeroth Order) } \\
\text { Circular A rc }\end{array}$ & 0.85 & 0.85 & & 9.1063 (exact) & 9.200 \\
$\begin{array}{l}\text { Parabolic } \\
\text { Arc I }\end{array}$ & 1.394 & 0.3011 & 0.645 & 8.412 & 7.937 \\
$\begin{array}{l}\text { Parabolic } \\
\text { Arc II }\end{array}$ & 1.232 & 0.4356 & 0.487 & 8.570 & 8.467 \\
\hline \hline
\end{tabular}

ing for $\omega_{1}$, we obtain

$$
\begin{aligned}
\omega_{1}= & -\frac{1}{2} \omega_{0} n^{2} \pi^{2}+R_{0}^{-3} \omega_{0}^{-1} \int_{0}^{1} \sin n \pi s\left[2 R_{1} n^{2} \pi^{2} \sin n \pi s\right. \\
& \left.-4 n \pi R_{1, s} \cos n \pi s-2 R_{1, s s} \sin n \pi s\right] d s .
\end{aligned}
$$

The solvability condition Eq. (23) gives the first-order correction to the nondimensional, natural frequency due to the effect of rotary inertia and variation of curvature. It is of importance to note that we do not have to solve the first-order equation per se to obtain $\omega_{1}$.

\section{RESULTS AND CONCLUSIONS}

To check the accuracy of the perturbation approximation, we calculated the natural frequencies of various circular and parabolic arcs and compared these calculated frequencies with their exact values and values obtained by using a finite element solution. The accuracy of the results depends on how small $\epsilon R_{1}(s)$ is compared with $R_{0}$.

As an initial example, the approximate fundamental natural frequencies of three circular arcs are compared with their exact solutions. An arc of nondimensional radius $R_{0}=1.072$ and $I / A L^{2}=0.001$ is considered as the zeroth-order problem. Then the radius is increased by about $10 \%, 20 \%$, and $30 \%$, and the perturbation solutions are obtained. In this case, $R_{1}(s)$ is a constant and the integral in Eq. (23) can be easily evaluated. The exact solutions are obtained from Eq. (19). The results are shown in Table $\mathrm{I}$.

As further examples, the fundamental natural frequencies of two parabolic arcs (Fig. 2) are computed using the first-order perturbation approximation. Here, $R_{0}$ for the arc is chosen such that it is approximately the mean radius or that

$$
\int_{0}^{1} R_{1}(s) d s \approx 0
$$

This would seem to contribute to a better accuracy of the results. Here, $I / A L^{2}$ for the arcs is 0.001 . Since $R_{1}(s)$ is not a constant, the integral in Eq. (23) cannot be evaluated in closed form. It is computed using a second order Runge-Kutta algorithm. For comparison, the natural frequencies of the parabolic arcs are obtained using a finite-element formulation. A 40 straight beam-element model is chosen for the finite element approximation. The beam element has a cubic displacement field and includes the effect of rotary inertia. The results are shown in Table II.

It is of interest to note that for the parabolic arcs, in spite of the radius of curvature deviating up to $65 \%$ from the mean (zeroth-order) value, the perturbation solution is within $6 \%$ of the finite-element solution. The present investigation effectively illustrates the ability of perturbation methods to obtain extremely good approximations. The analysis could be easily extended to beams with other boundary conditions and also to curved plates and shells.

${ }^{1}$ E. Volterra and J. D. Morell, "Lowest natural frequencies of elastic hinged arcs," J. Acoust. Soc. Am. 33, 1787-1790 (1961).

${ }^{2}$ E. Romanelli and P. A. Laura, "Fundamental frequencies of non-circular, elastic hinged arcs," J. Sound Vib. 24, 17-22 (1972).

${ }^{3}$ A. H. Nayfeh, Perturbation Methods (Wiley-Interscience, New York, 1973).

${ }^{4} \mathrm{~J}$. T. Oden, Mechanics of Elastic Structures (McGraw-Hill, New York, 1967).

${ }^{5}$ A. H. Nayfeh, "Nonlinear traverse vibrations of beams with properties that vary along the length," J. Acoust. Soc. Am. 63, 766-770 (1973). 